\title{
Le parrainage \\ « un chercheur, un enseignant, une classe »
}

\section{L'élaboration d'un partenariat durable entre des classes de lycées et l'université dans l'académie de Rouen}

Depuis 2005, l'université de Rouen s'est engagée, en partenariat avec le rectorat et le CNRS, dans la conception d'ateliers scientifiques à destination d'élèves d'écoles primaires et de collèges et lycées. Afin d'étoffer notre partenariat avec les lycées, nous avons élaboré, depuis deux ans, un parrainage entre une classe de lycée, son enseignant et un chercheur d'un laboratoire de l'université. Ce dispositif vise à faire découvrir, sur une période plus longue, le monde de la recherche et de l'université par des rencontres régulières entre le chercheur et la classe, tantôt au lycée, tantôt à l'université.

Désaffection pour les études scientifiques ou préférence pour les filières professionnelles?

La question de la " désaffection des jeunes pour les études scientifiques ", abondamment débattue depuis une bonne dizaine d'années, en particulier dans les pays développés, prend un sens très particulier en France en raison de son système dual d'enseignement supérieur universités/grandes écoles. En effet, la baisse avérée du nombre d'étudiants inscrits dans les filières scientifiques généralistes de l'université doit être examinée conjointement avec les autres cursus scientifiques.

Ainsi, on constate que d'autres filières scientifiques continuent à attirer un grand nombre d'étudiants comme, par exemple, les classes préparatoires et les écoles d'ingénieurs, les études médicales, les IUT qui relèvent du secteur secondaire, ainsi que bon nombre de classes de STS (sections de techniciens supérieurs).

Plutôt que de "désaffection pour les sciences ", peut-être vaudrait-il mieux parler de choix prioritaires des étudiants pour les filières professionnelles [1].

1 B. Convert, Les impasses de la démocratisation scolaire - Sur une prétendue crise des vocations scientifiques, Raisons d’Agir Éditions (2006).
À l'occasion de l'opération « 2005 : Année Mondiale de la Physique ", nous avons choisi, dans l'académie de Rouen, d'initier un partenariat durable entre les établissements scolaires du $1^{\mathrm{er}}$ et du $2^{\text {nd }}$ degré et l'université. Nous sommes partis du constat qu'un certain nombre des difficultés auxquelles sont confrontées les universités pour ce qui concerne "la désaffection des jeunes pour les études scientifiques " (voir l'encadré) provient de la méconnaissance de ce qu'est le monde de l'université, et plus particulièrement de ses spécificités liées à la recherche. Il nous a donc semblé nécessaire de développer, chez les élèves du second degré, une ouverture vers ce monde, et celui de la recherche en particulier.

\section{Des salles de sciences à l'université de Rouen}

Nous avons ainsi élaboré un dispositif permettant, d'abord dans le domaine de la physique, de tisser des relations entre des établissements scolaires et l'université. Ce dispositif a consisté, dans un premier temps, en l'aménagement de salles de science à l'université, pour permettre à des élèves de différents niveaux de participer à des ateliers scientifiques. Ces salles de science sont au nombre de quatre aujourd'hui : trois en physique et une en chimie. La visite d'une classe à l'université consiste typiquement en une demi-journée d'activités sous forme de deux ateliers successifs, que les élèves suivent en groupes de 10 à 15, suivis d'une conférence pour la classe entière sur des thèmes comme l'infiniment grand et l'infiniment petit, ou l'astronomie. Certains ateliers sont constitués d'expériences spectaculaires, impossibles à réaliser dans le cadre scolaire habituel. Il s'agit, par exemple, d'observations en microscopie électronique à balayage (fig. 1), d'une expérience permettant de réaliser un spray utilisé dans les moteurs à injection et où l'on injecte un jet 

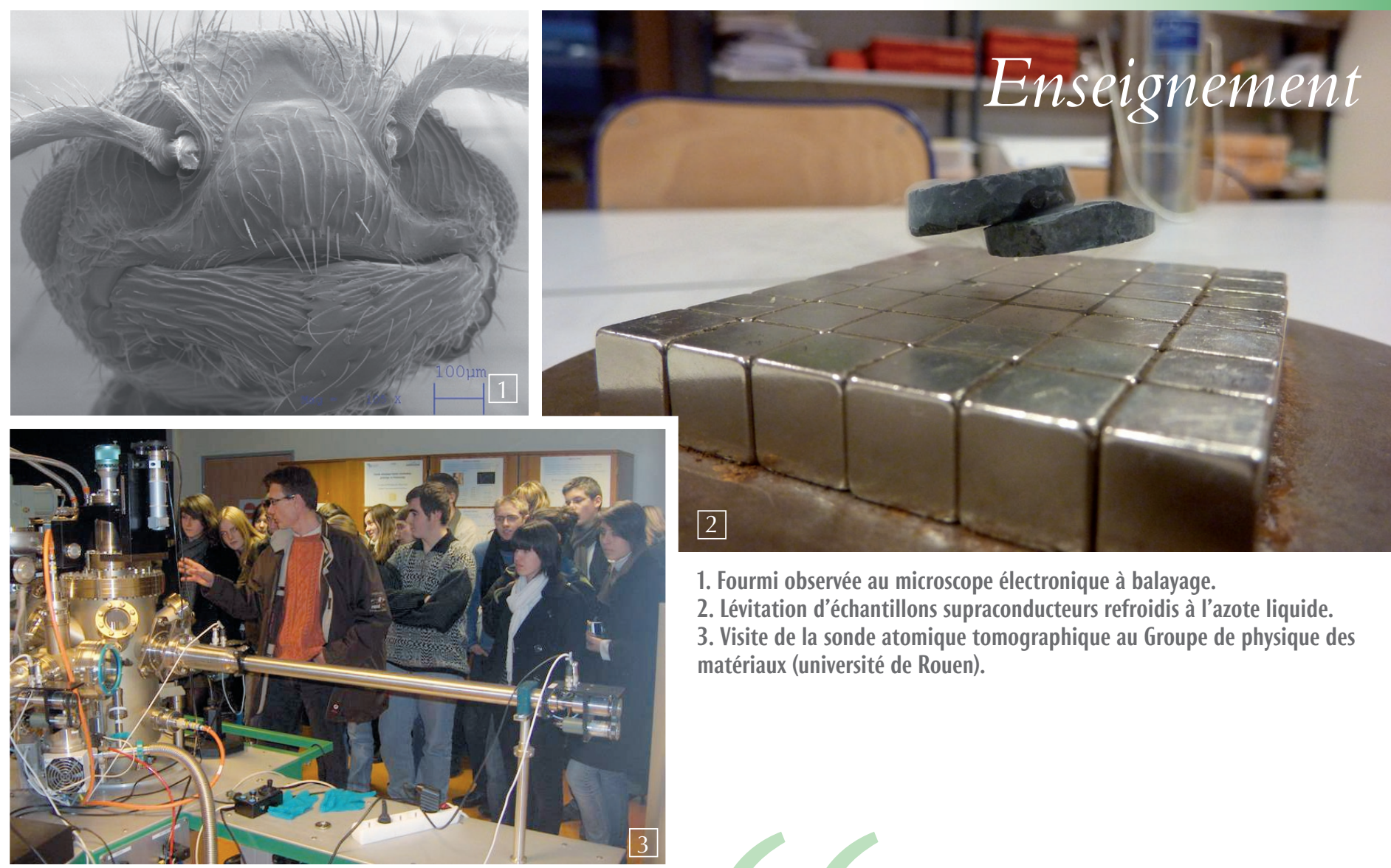

1. Fourmi observée au microscope électronique à balayage.

2. Lévitation d'échantillons supraconducteurs refroidis à l'azote liquide.

3. Visite de la sonde atomique tomographique au Groupe de physique des matériaux (université de Rouen).

d'air comprimé dans un jet d'eau, ou encore de la lévitation d'un matériau supraconducteur refroidi à l'azote liquide au-dessus d'un damier d'aimants permanents (fig. 2)...

Pour les élèves de lycées, nous concentrons les activités proposées autour des thématiques de recherche présentes sur le site, en organisant des visites de laboratoires (fig. 3), pour l'essentiel le Groupe de physique des matériaux (UMR CNRS 6634) et le CORIA (UMR CNRS 6614).

L'ensemble de ce dispositif, qui nous permet d'accueillir chaque année 40 à 45 classes d'écoles élémentaires de cycle 3 (CE2CM1-CM2) et 30 à 40 classes de collèges et lycées de l'Académie, a pu voir le jour grâce à la signature d'une convention tripartite, reconduite annuellement, entre le rectorat de l'académie de Rouen, la délégation Nord-Ouest du CNRS et l'université de Rouen. Dans le cadre de cette convention, la participation du CNRS concerne les équipements des salles de sciences (environ 1500 euros par an). Le rectorat et l'université, quant à eux, s'engagent par la mise à disposition de personnels pour mener à bien cette opération. Ainsi, pour ce qui concerne l'université, un équivalent temps plein enseignement d'un enseignant-chercheur (192 heures) est affecté à ce dispositif, à moitié pour le $1^{\text {er }}$ degré et à moitié pour le $2^{\text {nd }}$ degré. Il nous a semblé, dès le départ, que cette prise en compte effective dans le service d'enseignement des enseignantschercheurs était la condition nécessaire pour permettre une pérennisation du dispositif, au-delà de la simple bonne volonté que permettait la visibilité de l'opération «2005: Année Mondiale de la Physique ». Une dizaine d'enseignants-chercheurs, dont trois doctorants, participent aux animations de ces ateliers et visites de laboratoires, ce qui permet d'éviter de concentrer l'essentiel des activités sur deux ou trois collègues «spécialistes".

Interview de Philippe Pareige, professeur de physique à l'université de Rouen, directeur-adjoint du Groupe de physique des matériaux, et co-responsable d'un parrainage " un chercheur, un enseignant, une classe ".

Pour quelles raisons avez-vous choisi de participer à cette opération de parrainage avec une classe de lycée et son enseignant?

J'ai participé, pendant de nombreuses années, aux opérations de diffusion de la culture scientifique, telles que la Fête de la Science. Ce qui m'a plus particulièrement intéressé dans ce nouveau dispositif c'est, d'une part, la proximité avec les élèves et la possibilité d'établir un lien durable avec eux, d'autre part, la liberté de choisir le sujet et de permettre aux élèves de prendre conscience qu'il y a un lien entre le domaine de la recherche et le monde dans lequel ils vivent.

Avez-vous le sentiment que des élèves de lycée soient réceptifs à la présentation des problématiques, souvent complexes, rencontrées par les chercheurs dans leurs laboratoires?

Absolument. Mais, bien entendu, cela nécessite du travail en amont pour préparer les séances avec les élèves. À titre d'exemple, nous avons travaillé l'année dernière sur les nanosciences dans le domaine de la téléphonie mobile. J'ai conçu une séance, au cours de laquelle j'ai démonté pièce par pièce un téléphone portable, puis fait une présentation à l'aide d'un diaporama des caractéristiques des matériaux utilisés dans les différentes générations d'appareils. Ce genre de séance ne peut évidemment pas s'improviser.

Pensez-vous que cette expérience est susceptible d'améliorer la perception qu'ont les élèves des sciences en général ?

Oui, pour deux raisons à mon avis. Premièrement, parce qu'elle permet aux élèves de connaître les métiers de la recherche et certains de ses acteurs (les doctorants, les post-docs, souvent étrangers, par exemple). Deuxièmement, en montrant aux élèves que la recherche n'est pas une activité isolée du reste de la société, mais qu'elle interagit constamment avec elle. 


\section{\〉> \\ Le parrainage « un chercheur, un enseignant, une classe »}

Depuis deux ans, nous avons élargi notre offre de partenariat, avec les lycées en particulier, en essayant de susciter une relation plus soutenue qu'une simple visite d'une demi-journée. Ainsi, nous avons initié une opération de parrainage intitulée "un chercheur, un enseignant, une classe ", qui consiste en des rencontres échelonnées tout au long de l'année scolaire entre un enseignant-chercheur, une classe de lycée et son enseignant. Les rencontres, au nombre de quatre au minimum au cours de l'année scolaire, ont lieu tantôt à l'université, tantôt au lycée. Elles fournissent au chercheur l'occasion de présenter son parcours, son métier au quotidien, l'élaboration et la mise en œuvre d'un projet de recherche sur le long terme. Les visites à l'université permettent aux élèves de découvrir l'univers de la recherche, la réalité du travail dans les laboratoires, les parcours et les motivations des enseignants-chercheurs. Ce parrainage doit favoriser l'intérêt des élèves pour les études scientifiques et doit leur permettre également de découvrir les métiers liés à la recherche.

En 2008-2009, 8 projets de parrainage impliquant 8 chercheurs et 8 classes de lycée $\left(2^{\text {nde }}, 1^{\text {re }} \mathrm{S}\right.$, Terminale $\left.\mathrm{S}\right)$ ont ainsi été menés à leur terme, tous dans le domaine de la physique (nanosciences, matériaux magnétiques artificiels, métrologie optique, combustion, matériaux polymères). À titre d'exemple, des élèves de $1^{\text {re }} \mathrm{S}$ du lycée Vallée du Cailly ont participé à la conception d'un banc expérimental de métrologie optique; d'autres lycéens ont participé, sur la base du volontariat, à une demi-journée de manipulations pour la réalisation de multicouches magnétiques. À la suite de cette première expérience, il nous a semblé utile de susciter plutôt la participation de classes de $1^{\text {re }} \mathrm{S}$, dont le niveau paraît plus adapté au déroulement du projet scientifique tout au long de l'année.

Devant le succès de ce dispositif (voir les interviews, pp. 27-28), le rectorat a souhaité que nous élargissions notre offre de partenariat, en couvrant d'autres disciplines scientifiques. Ainsi, 25 projets impliquant 25 enseignants-chercheurs ou chercheurs des universités de Rouen et du Havre ont été menés à bien avec une vingtaine de lycées de l'Académie, en 2009-2010. Ils ont concerné la physique, la chimie, la biologie, les mathématiques, l'informatique et la bio-informatique. Ces projets sont conduits avec une grande liberté par les binômes constitués d'un chercheur et de son homologue enseignant en lycée. Ils peuvent même permettre une interaction avec des étudiants de licence par exemple, comme cela a été le cas l'an dernier sur un projet de cryptographie informatique, pour lequel les étudiants de troisième année de licence d'informatique ont utilisé les lycéens comme " cobayes " pour tester les programmes mis au point dans le cadre de leur projet. On a alors pu constater que les lycéens avaient une relation beaucoup plus spontanée avec des étudiants, de quelques années leurs aînés, qu'avec des enseignants ou des chercheurs, ce qui peut contribuer à leur donner une image moins impersonnelle du milieu universitaire.

Pierre-Emmanuel Berche (pierre.berche@univ-rouen.fr) Groupe de Physique des Matériaux, UMR CNRS 6634, Université de Rouen, B.P. 12, 76801 Saint-Étienne du Rouvray Cedex

Interview de Christophe Lemonnier, professeur agrégé de sciences physiques au lycée Gustave Flaubert de Rouen

Vous participez, depuis deux ans maintenant, au projet de parrainage "un chercheur, un enseignant, une classe ", en partenariat avec un professeur de l'université de Rouen. Qu'est-ce qui vous a motivé à participer à cette opération?

D'un point de vue personnel, j'ai moi-même suivi des études universitaires et j'ai eu envie de renouer un contact avec l'université et ses enseignants. Sur un plan plus professionnel, j'ai la conviction que les élèves de lycée ignorent la plupart du temps le contenu de ces études et je souhaitais le leur faire découvrir. Par ailleurs, je considère que l'enseignement de physique-chimie au lycée permet de poser les fondamentaux qui trouvent parfois un prolongement dans la vie courante. Ce projet permet d'appréhender ce prolongement, en constatant que la physique sort du cadre purement scolaire, de ce qui est vu au lycée, pour être mise en pratique au travers de la recherche scientifique et de ses applications.

\section{Avec quelles classes avez-vous travaillé ?}

Des classes de $1^{\text {re }} \mathrm{S}$, ce qui me semble le plus approprié car les élèves ont déjà choisi de faire des sciences. En classe de $2^{\text {nde }}, c^{\prime}$ est vraisemblablement plus compliqué car on se heurte alors au blocage d'une certaine proportion des élèves.

Avez-vous le sentiment que le fait de participer à cette action change le regard de certains élèves par rapport à la poursuite de leurs études ou à leur éventuel projet professionnel? Cela influence-t-il l'ambiance de travail dans la classe?

Compte tenu de mon expérience, j'ai le sentiment que le projet de parrainage constitue une aide certaine au choix d'orientation en fin de $1^{\text {re }} S$ entre les spécialités mathématiques, physique-chimie ou sciences de la vie et de la terre (SVT). Habituellement, les deux tiers des élèves s'orientent vers la spécialité SVT, les autres se répartissant entre maths et physique-chimie. L'année dernière, dans la classe que j'ai parrainée, une vingtaine d'élèves sur trente-cing ont fait le choix de la spécialité physique-chimie. Je pense que le parrainage a incontestablement influencé ce choix. En ce qui concerne leur éventuel projet professionnel, la plupart des élèves n'imaginent pas qu'un ingénieur peut travailler dans le domaine de la recherche, et que le fait de poursuivre des études longues permet d'envisager une plus grande diversité de métiers. Quant à l'ambiance de travail dans la classe, je pense que le fait de participer à ce projet donne un surcroît de crédibilité à l'enseignant, en montrant aux élèves que les savoirs scientifiques enseignés en classe sont en prise avec la réalité. L'implication des élèves dans ce projet est évidente et se mesure au fait que lorsque je leur demande de faire des comptes rendus à la suite des visites, les élèves y participent toujours de façon volontaire. Sur trente-cinq élèves, seule une petite minorité ne s'implique pas dans la mise en œuvre du projet. J'ai vraiment le sentiment que la participation à cette opération facilite le lien avec la classe dans l'enseignement au quotidien.

Sur quels projets avez-vous travaillé dans le cadre de ces parrainages?

Les projets sur lesquels nous avons travaillé avec Philippe Pareige sont définis dans le cadre d'une discussion avec les élèves, à la suite d'une présentation par M. Pareige des différents thèmes qui peuvent être abordés. La première année, cela a concerné l'énergie et le développement des matériaux, en particulier dans le domaine du nucléaire. Le projet a permis l'organisation d'une visite d'une journée au CEA à Saclay. L'année dernière, les élèves avaient le choix entre les applications des nanomatériaux dans trois domaines différents : le domaine médical, les applications pour la téléphonie mobile ou pour le nucléaire. Ils ont choisi le second thème ; nous avons donc travaillé sur les notions de transistors et de propriétés des semi-conducteurs, et avons organisé une visite au CNRT Matériaux de Caen. Il convient de noter l'effort important fourni par les chercheurs pour la vulgarisation de leurs présentations à nos élèves. 